\title{
SELF-GRAVITATING POLAR RING MODELS
}

\author{
Linda S. Sparke \\ Institute of Astronorny, Cambridge, U.K.
}

In a number of SO galaxies, rings of gas, dust and stars are observed to lie roughly perpendicular to the galactic disc (e.g. Whitmore, these proceedings). The material sornetirnes forms a fairly broad annulus, and the ring appears nearly flat. Sirnple estimates suggest that differential precession will destroy a polar ring in much less than a Hubble tirne, irnplying that the observed structures have been formed only recently. It is then surprising that some of them are so regular, unless sornething acts to prevent their disruption.

A simple rnodel for a self-gravitating polar ring is a collection of concentric but mutually tilted massive circular wires, in orbit about a flattened potential representing the body of the SO galaxy. Equilibrium states can be found in which the wires precess steadily as a unit, making up a coherent polar ring. There are stable equilibria near the pole provided that the ratio of the ring mass to the galactic quadrupole rnornent exceeds a minimurn value (which increases as the ring becornes broader). Stable polar rings are nearly planar, but curve up towards the pole at their outer edge. This is the opposite sense of warping from that expected in a polar ring which is stabilised in orbit about the short axis of a rotating triaxial figure; these rings bend towards the equator (van Albada et al. 1982; MNRAS 198, 303). The required masses are cornparable to those observed in neutral hydrogen (e.g. Schechter et al. 1984; MNRAS 208, 111).

Unless the polar ring is extremely heavy, stable states exist only near the pole or near the equatorial plane; rings at intermediate angles are unstable and will break up. Time-dependent calculations show that unstable rings split into a number of stable and independently precessing sub-rings. Thus a multiple polar ring system (such as that in ESO 474-G20: Schweizer et al. 1983; A.J. 88, 909) may result from capturing a single gas cloud.

A full account of this work will appear in Monthly Notices of the Royal Astronomical Society. 